Kardiologe 2020 $14: 155$

https://doi.org/10.1007/s12181-020-00379-z

Online publiziert: 13 . Februar 2020

(c) Springer Medizin Verlag GmbH, ein Teil von Springer Nature 2020

\section{Originalpublikation}

Mackay DF, Russell ER, Stewart K et al (2019) Neurodegenerative disease mortality among former professional soccer players. New Engl J Med 381:1801-08

Ein sportassoziierter plötzlicher Herztod tritt vermeintlich besonders häufig im Fußball auf, was allerdings mehr dessen gesellschaftlicher Popularität und Medienpräsenz als der statistischen Realität geschuldet ist. Doch wie sieht es eigentlich langfristig mit der Gesundheit von Profi-Fußballern aus? Neue Daten aus Schottland geben hierzu jetzt interessanten Aufschluss.

\section{Zusammenfassung der Studie}

Analysiert wurden Todesursachen ehemaliger schottischer Profifußballer ( $n=7676$; Alter $>40$ Jahre) sowie einer altersgematchten Kontrollpopulation über einen Zeitraum von 18 Jahren; zusätzlich erfasst wurde die Häufigkeit einer antidemenziellen Medikation. Insgesamt starben vergleichbar viele Personen beider Gruppen (15,4\% vs. 16,5\%). Bis zum Alter von 70 Jahren ergab sich dabei zunächst ein Überlebensvorteil für Fußballer, danach wendete sich allerdings das Blatt: Zwar starben weniger Ex-Profis an den Folgen einer ischämischen Herzkrankheit (Hazard Ratio 0,80 [95\%-KI(Konfidenzintervall) 0,66-0,97]; $p=0,02)$, doch zeigte sich eine signifikant höhere Mortalität durch neurodegenerative Erkrankungen (1,7\% vs. $0,5 \% ; 3,45$ [2,11-5,62]; $p<0,001)$. Dies betraf vorwiegend Fußballer mit Alzheimer-Erkrankung, weniger mit Parkinson. Entsprechend erhielten Fußballer fast 5-mal häufiger eine Demenz-

Axel Preßler

Sportkardiologie München, München, Deutschland

\title{
Fußball ist Kopf-, nicht Herzenssache!
}

medikation als Nichtsportler, darunter signifikant weniger Torhüter als Feldspieler.

\section{Kommentar}

Gemäß den hohen Spielerzahlen bis hin in den Breitensport ist auch in Deutschland die Zahl plötzlicher Todesfälle im Fußball am höchsten [1], wenngleich absolut gesehen sehr gering. Ausreichende Evidenz aus randomisierten Studien für einen präventiven Effekt fußballerischer Aktivität auf kardiovaskuläre Risikofaktoren gibt es mangels geeigneter Studien bisher nicht [2]. Die vorliegende epidemiologische Arbeit spricht aber zumindest für einen langfristigen Schutz vor Herzerkrankungen, was der allgemein erhöhten Lebenserwartung ehemaliger Leistungssportler entspricht - sportartbedingte Unterschiede sind hier allerdings nicht auszuschließen.

Nicht zuletzt durch den „Knockout“ des Nationalspielers Christoph Kramer im WM-Finale 2014 erfuhr die chronisch-traumatische Hirnschädigung (engl. „concussion“) durch repetitive Kopfbälle oder -kollisionen zuletzt viel mediale und wissenschaftliche $\mathrm{Be}$ achtung. Untersuchungen liegen v. a. für ehemalige Spieler des kollisionsträchtigen American Football vor, die ebenfalls erhöhte Raten neurologischer Spätfolgen sowie auch konkrete neurodegenerative Veränderungen aufweisen [3, 4]. Als Prophylaxe gegenüber diesem „Berufsrisiko" wird bereits eine Helmpflicht für Fußballer diskutiert, zudem wurden zur laufenden Saison erstmals verpflichtende neurologische Baseline-Screenings für Erst- und Zweitligavereine in Deutschland eingeführt.
Welche Schlüsse sollte man also aus den Daten ziehen? Dem Klischee nach scheuen schottische Fußballer den harten Zweikampf nicht, aber auch in Deutschland gilt ein sauber ausgeführter Kopfball seit jeher als hohe Kunst des Fußballspielens, daher darf man kaum auf Länderunterschiede verweisen. Dennoch weitere Analysen sind sicher notwendig, besonders im Hinblick auf den Breitensport und auf Frauen, bevor man das Wort „Fußball“ allzu wörtlich nimmt und Kopfbälle gar verbietet. Bis dahin sollten Spieler, Eltern und Trainer dem vermutlich weitaus vorteilhafteren Effekt regelmäßiger Bewegung auf dem Fußballfeld vertrauen.

\section{Korrespondenzadresse}

PD Dr. med. Axel Preßler

Sportkardiologie München

Törringstr. 6, 81675 München, Deutschland

axel.pressler@kardiologie-mit-herz.de

Interessenkonflikt. A. Preßler gibt an, dass kein Interessenkonflikt besteht.

\section{Literatur}

1. Bohm P, Scharhag J, Meyer T et al (2016) Data from a nationwide registry on sports-related sudden cardiac death in Germany. Eur J Prev Cardiol 23:649-656

2. Eberl M, Tanaka LF, Klug SJ et al (2019) Football as a health promotion strategy - a systematic review of football intervention studies. Dtsch Arztebl Int 116:721-728

3. Montenigro PH, Alosco ML, Martin BM et al (2017) Cumulative head impact exposure predicts laterlife depression, apathy, executive dysfunction, and cognitive impairment in former high school and college football players. J Neurotrauma 34:328-340

4. Stern RA, Adler CH, Chen Ketal (2019) Tau positronemission tomography in former national football league players. N Engl J Med 380:1716-1725 